\title{
Study on microcrystalline cellulose/chitosan blend foam gel material
}

https://doi.org/10.1515/secm-2020-0047

Received May 12, 2020; accepted Nov 11, 2020

\begin{abstract}
In the present contribution, an environmentalfriendly and cost-effective adsorbent was reported for soil treatment and desertification control. A novel foam gel material was synthesized here by the physical foaming in the absence of catalyst. By adopting modified microcrystalline cellulose and chitosan as raw materials and sodium dodecyl sulfonate (SDS) as foaming agent, a microcrystalline cellulose/chitosan blend foam gel was synthesized. It is expected to replace polymers derived from petroleum for agricultural applications. In addition, a systematical study was conducted on the adsorbability, water holding capacity and re-expansion performance of foam gel in deionized water and brine under different SDS concentrations $(2 \%-5 \%)$ as well as adsorption time. To be specific, the adsorption capacity of foam gel was up to $105 \mathrm{~g} / \mathrm{g}$ in distilled water and 54g/g in brine, indicating a high water absorption performance. As revealed from the results of Fourier transform infrared spectroscopy (FTIR) analysis, both the amino group of chitosan and the aldehyde group modified by cellulose were involved. According to the results of Scanning electron microscope (SEM) analysis, the foam gel was found to exhibit an interconnected pore network with uniform pore space. As suggested by Bet analysis, the macroporous structure was formed in the sample, and the pore size ranged from 0 to $170 \mathrm{~nm}$. The mentioned findings demonstrated that the foam gel material of this study refers to a potential environmental absorbent to improve soil and desert environments. It can act as a powerful alternative to conventional petroleum derived polymers.
\end{abstract}

Keywords: Microcrystalline cellulose-chitosan; Water absorption; Modification; Foam gel

\footnotetext{
*Corresponding Author: Hongkai Zhao: School of Materials Sci-

*Corresponding Author: Hongkai Zhao: School of Materials Sci-
ence and Engineering, Jilin Jianzhu University, Changchun 130118, China; Email: hkzhao003@126.com; Tel.: +86-0431-13596077798 Kehan Zhang, Shoupeng Rui, Peipei Zhao: School of Materials Science and Engineering, Jilin Jianzhu University, Changchun 130118, China
}

๖ Open Access. ๑ 2020 H. Zhao et al., published by De Gruyter. (cc) BY License

\section{Introduction}

The continuous expansion of soil desertification endangers humans and ecosystems, while resulting in serious environmental pollution. It has aroused rising attention over the past few years [1]. Accordingly, environmental-friendly water absorbents are urgently required.

Chitosan and cellulose pertain to the category of the most abundant biomass resources worldwide. As polymer biosorbents, the two have received growing attention for their abundant raw materials [2], variable functional groups, and similarities in chemical structures. Cellulose based biosorbents can be synthesized from cellulose and its derivatives with a range of methods, whereas they exhibit several defects (e.g., poor film-forming ability). Among a considerable number of biopolymers, chitosan exhibits high hydrophilicity, antibacterial properties, biodegradability and biocompatibility. It is synthesized by deacetylation based on chitin. Since the chain is abundant in hydroxyl and amino groups [3-5], chitosan has received extensive studies in numerous fields (e.g., biomedicine and water treatment) [6-11]. In addition, blended polymers exhibit more advantages than singlecomponent materials and can remedy the defects of singlecomponent materials. As indicated by existing studies, MCC/CS blends retain the water absorption of cellulose, while improving the moisture absorption and antibacterial properties of chitosan. Recently, MCC/CS based biodegradable absorbent materials have suggested significant environmental and economic benefits, and they have become the research hotspots.

Chemical cross-linking refers to a permanent connection formed by covalent bonds between polymers, commonly improving the mechanical properties of polymers. Overall, conventional water-absorbing materials are synthesized in the presence of cross-linking agents (e.g., acrylic acid, acrylamide, glutaraldehyde, polyethylene glycol, potassium persulfate and tripolyphosphoric acid) [1215]. Via the chemical cross-linking, permanent connection can be developed by covalent between polymers, generally improving the mechanical properties of materials. Ferfera Harrar et al. [16] reported a chitosan grafted poly- 
acrylamide and carboxymethylcellulose based water absorbent material exhibiting high water retention performance, which achieved a maximum water absorption of $562 \mathrm{~g} / \mathrm{g}$, as well as a rather high absorption of $421 \mathrm{~g} / \mathrm{g}$ after several cycles of expansion. To enhance the strength of hydrogel more significantly, researchers have conducted numerous studies on modified chitosan. Wang et al. [17] synthesized hydrogels by employing trimethyl chitosan and sodium carboxymethylcellulose as the matrix and epoxypropyltrimethyl ammonium chloride as the etherifying agent. As revealed from a further characterization, the tensile strength of product has been significantly enhanced.

Chemical modification of cellulose and chitosan (e.g., esterification [18], etherification [19], cross-linking and grafting [20]) significantly improved their water absorption capacity. Essawy et al. [21] synthesized a soil waterretaining agent by the graft copolymerization of cellulose/chitosan by applying acrylic acid as the crosslinking agent. Under the grafting ratio of $86.4 \%$, the maximum water absorption was achieved. However, the raw materials of synthetic polyacrylic acid originate from non-renewable resources (e.g., petroleum), resulting in the defects of secondary pollution. Recently, people have generated an increasing interest in producing the water absorbent materials via physical cross-linking due to the relatively simple production and non-requirement of cross-linking agents. A variety of physical interactions between molecules have been studied to inspire the design of novel water absorbent materials, the formation of 3D composites and ionic interactions, etc. [22-27]. However, the reported cellulosebased water absorbent materials produced via physical cross-linking thus far have exhibited poor biodegradability and low absorption capacity.

To tackle down the mentioned challenges, a novel preparation method has been proposed in this study. With MCC/CS as the carrier, a new foam gel was generated by adding SDS in the absence of catalysts. The cross-sectional morphology and swelling behavior of the crosslinked foam gel were studied by performing a series of experiments. On that basis, a novel prospect for the preparation of foam gel with excellent properties was presented.

\section{Materials and methods}

\subsection{Materials}

Chitosan (CS, Deacetylation degree 97\%) was provided by Huibaiwei biotechnology Co., Ltd(Henan, china) with an average particle size of 4.07um. Microcrystalline cellulose was offered by Qingjun building materials Co., Ltd (Shandong, China), exhibiting an average particle size of 90um. Sodium periodate $\left(\mathrm{NaIO}_{4}\right), \mathrm{NaCl}$, anhydrous ethanol, isopropyl alcohol, SDS, and chloroacetic acid were all purchased from Jilin Haodi Reagent Distribution Co, Ltd (Jilin, China). All chemical reagents were of analytical grade, and all solutions were synthesized with deionized water.

\subsection{Preparation of foam gel}

The synthesis of DAMCC/CMCS foam gel consisted of the following three steps (Figure 1). First, MCC (10g) was added to $500 \mathrm{ml}$ deionized water, where $10 \mathrm{~g} \mathrm{NaIO}_{4}$ and $10 \mathrm{~g} \mathrm{NaCl}$ were introduced for oxidation. The resulting solution was stirred at ambient temperature $\left(25^{\circ} \mathrm{C}\right)$ for 8 $\mathrm{h}$ in dark, cleaned by centrifugation and subsequently dried to obtain modified cellulose (DAMCC). Next, CS (1g) was dissolved in the mixed solution of $\mathrm{NaOH}(1 \mathrm{~g})$, isopropanol $(48 \mathrm{ml})$ and water $(12 \mathrm{ml})$ for volume expansion. Then, the upper CS slurry was added to the mixture of chloroacetic acid (1.5g) and isopropanol (2ml), which was continuously stirred for $12 \mathrm{~h}$. After being washed and dried, carboxymethyl chitosan (CMCS) was synthesized. Lastly, DAMCC, CMCS and SDS were mixed at different ratios and then stirred at a high speed of $4000 \mathrm{rpm} / \mathrm{min}$ for $5 \mathrm{~min}$. The resultant mixture was heated at $60^{\circ} \mathrm{C}$ for $1 \mathrm{~h}$ and then dried
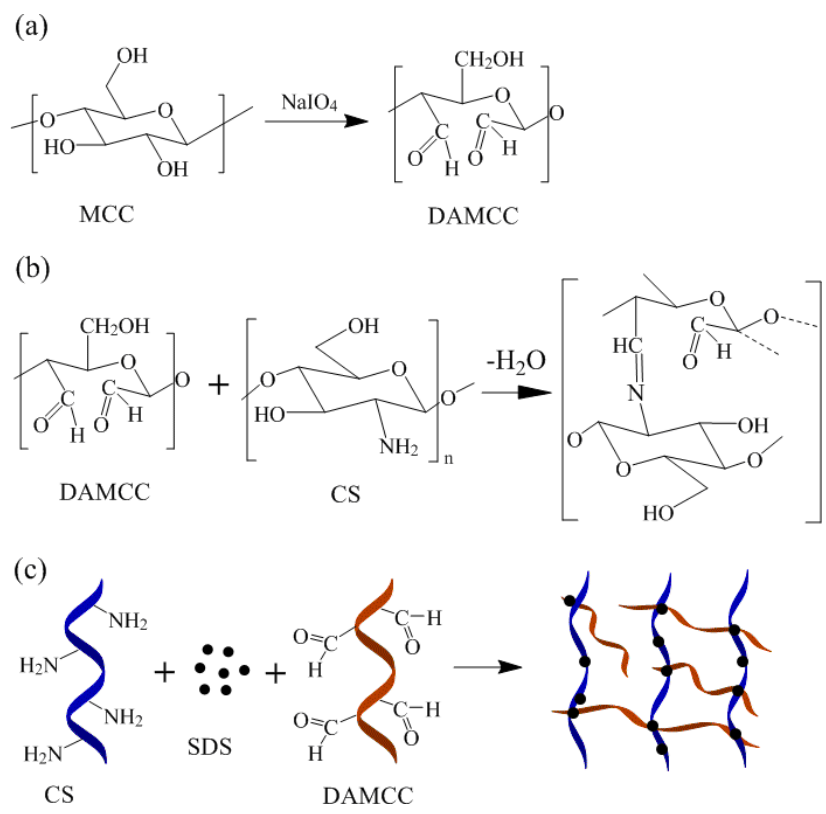

Figure 1: (a) MCC oxidized to DAMCC by $\mathrm{NaOl}_{4}$; (b) Schiff base reaction between DAMCC and CS; (c) Synthesis of DAMCC/CMCS foam gel 
in vacuum freeze dryer for $72 \mathrm{~h}$ to produce lightweight porous foam gel. It is noteworthy that water is the only byproduct in the reaction. Moreover, the mentioned reaction required no expensive catalysts and reaction purification steps. Thus, the process is considered to be eco-friendly.

\subsection{Characterization}

\subsubsection{Determination of aldehyde group content in modified cellulose}

The hydrochloric acid released from the quantitative reaction of hydroxylamine hydrochloride and aldehyde group was titrated with $\mathrm{NaOH}$ aqueous solution, and the reaction formula is written as:

$$
-\mathrm{CHO}+\mathrm{NH}_{2} \mathrm{OH} \cdot \mathrm{HCl} \rightarrow \mathrm{CHNOH}+\mathrm{HCl}+\mathrm{H}_{2} \mathrm{O}
$$

The $\mathrm{pH}$ value of the solution was regulated to 5 with aqueous $\mathrm{NaOH}$. $0.5 \mathrm{~g}$ of DAMCC $(0.5 \mathrm{~g})$ was dissolved in $20 \mathrm{ml}$ of deionized water, in which $20 \mathrm{ml}$ of hydroxylamine hydrochloride $(0.05 \mathrm{~g} / \mathrm{ml})$ was introduced. The resultant solution underwent the $5 \mathrm{~h}$ stirring at $50^{\circ} \mathrm{C}$. Next, the $\mathrm{pH}$ value of above solution was adjusted with $0.1 \mathrm{~mol} / 1$ $\mathrm{NaOH}(\mathrm{pH}=5)$, and the consumed volume of $\mathrm{NaOH}(\mathrm{aq})$ was recorded. Likewise, MCC was titrated. Accordingly, the aldehyde content $(\mathrm{Q})$ was determined by [28]:

$$
Q=\frac{C_{1} \times\left(V_{1}-V_{2}\right)}{M_{1}}
$$

Where $\mathrm{Q}$ denotes the aldehyde content of $\mathrm{NaIO}_{4}$ modified MCC; $C_{1}$ represents the concentration of standard $\mathrm{NaOH}$ aqueous solution (moL/L); $V_{1}$ and $V_{2}$ are the volume of standard NaOH solution consumed by DAMCC and MCC, respectively; $M_{1}$ denotes the quality of DAMCC(g).

\subsubsection{Water absorption and water retention test}

Water absorption of foam gel was studied at $30^{\circ} \mathrm{C}$ and $50 \%$ relative humidity. The foam gel was first immersed in deionized water and in brine $(0.9 \mathrm{wt} \% \mathrm{NaCl})$, respectively. Subsequently, both samples were weighed at intervals. The water absorption (W) of foam gel was calculated:

$$
W=\frac{W_{t}-W_{d}}{W_{d}}
$$

Where $W$ denotes the water absorption rate of foam gel at time; $W_{t}$ represents the weight of wet material at time $t ; W_{d}$ is the weight of dry material $(\mathrm{g})$.

Moreover, the water-retention property of foam gel was determined at ambient temperature. The foam gel was first immersed in deionized water and brine, respectively, till the swelling equilibrium was reached. Next, the samples were taken out and placed in a drying box at $40^{\circ} \mathrm{C}$ to be ventilated and dried. Afterwards, both samples were weighted at intervals. The water retention value (WRV) was calculated by:

$$
W R V=\frac{M_{2}}{M_{3}} \times 100 \%
$$

Where $W R V$ denotes the water retention value of samples at a certain time; $M_{2}$ is the residual water absorption of samples at that time; $M_{3}$ represents the saturated water absorption of samples.

\subsubsection{Foam stability test}

The mixture was rapidly foamed at $4000 \mathrm{rpm} / \mathrm{min}$, and foam stability $(\mathrm{Fr})$ was determined by:

$$
F_{r}=R_{t}-T
$$

Where $F_{r}$ denotes the bubble height at time when the sample is bubbling; $R_{t}(\mathrm{ml})$ is the initial height of foam after foaming; $T(\mathrm{ml})$ represents the foam height at different time after foaming.

\subsubsection{Swelling properties of foam gel}

The re-expansion property of foam gel was determined by exploiting kinetics. $1 \mathrm{~g}$ of foam gel was soaked in water and then dried at ambient temperature. Subsequently, the water swelling property of foam gel was calculated by Formula 3. The mentioned soaking process acted as a reexpansion cycle, repeated four times.

In addition, the surface morphology of foam gel was characterized under the scanning electron microscope (SEM) (FEI Inspect 1450, Germany), exhibiting a high resolution of $3.0 \mathrm{~nm}$ under high vacuum as well as at an accelerating voltage of $30 \mathrm{KV}$. Fourier Transform infrared (FTIR) spectrum was detected to identify the functional groups of foam gel with the use of Bruker-Tensor 27 IR equipment (vertex 70, Brooke, Germany). By X-ray diffraction (XRD, D/max2500/PC, science company, Japan), the crystal structure of foam gel was analyzed. 


\section{Results and discussion}

\subsection{Analysis of aldehyde group content}

After hydroxylamine hydrochloride was introduced to DAMCC for a certain period, the solution color varied from white to light yellow. However, after the addition of hydroxylamine hydrochloride into to MCC, the color of the solution remained unchanged as white. It was therefore demonstrated that hydroxylamine hydrochloride reacted with aldehyde group in DAMCC. According to Formula 2, the aldehyde content was $(6.4 \pm 0.3) \mathrm{mmol} / \mathrm{g}$.

\subsection{Water absorption and water retention of foam gel}

\subsubsection{Water absorption analysis}

As revealed from the orthogonal test analysis, the effect of different factors on water absorption is: $\mathrm{C}>\mathrm{A}>\mathrm{B}$. To be specific, under the DAMCC content of $3 \%$, the CMCS content takes up $2 \%$, and under the SDS content of $4 \%$, the water absorption effect is improved. Since the orthogonal experiments represent only the optimal results for the conditions selected, they are subject to some limitations. By performing the mentioned orthogonal experiments, the effect of SDS concentration ( $2 \%-5 \%)$ on water absorption of foam gel was studied in depth.

Figure 2 illustrates the effect of different SDS concentrations on the deionized water absorption of foam gel. With the increase in SDS concentration, the deionized wa-

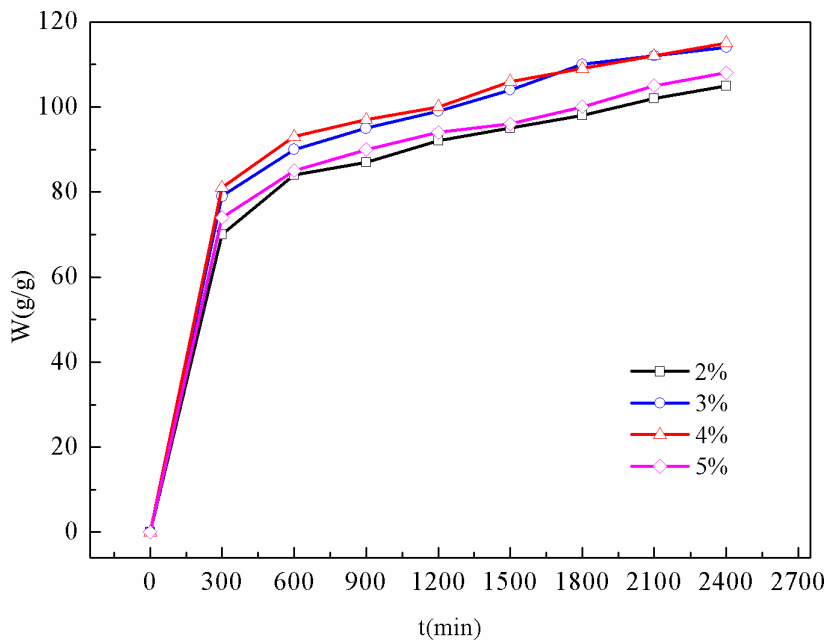

Figure 2: The deionized water absorption of foam gel at different SDS concentrations ter absorption was improved. At a concentration of $4 \%$, the deionized water absorption reached its peak. Under the concentration over $4 \%$, the deionized water absorption decreased. This result was attributed to the excessive foaming, thereby resulting in incomplete crosslinking of foams and gels. The deionized water absorption of foam gel could reach $70 \mathrm{~g} / \mathrm{g}$ rapidly and continued to increase. This was because the pore structure of foam gel was open and water entered rapidly. After 2 days of water absorption, the deionized water absorption rate of the foam gel could reach $(105 \pm 1.7) \mathrm{g} / \mathrm{g}$, and the integrity of its basic morphology could be maintained.

Figure 3 illustrates the effect of different SDS concentrations on the brine adsorption of foam gel. This figure indicates that the foam gel could absorb a maximum of $(54 \pm 2.0) \mathrm{g} / \mathrm{g}$ of brine, and its adsorption capacity was significantly lower than that of deionized water. This finding resulted from the existence of hydrophilic ion - $\mathrm{OH}$ in the network structure of the foam gel, while the charge shielding effect of cation $\left(\mathrm{Na}^{+}\right)$in the brine led to the reduction of the electrostatic repulsive force of the anion. It caused a decrease in osmotic pressure between the foam gel network and the external solution, thereby resulting in a reduction in the ability to absorb brine.

Based on mentioned results, the ability of the foam gel of this study to absorb distilled water remained lower than that of commercial gels, whereas it is worth noting that its ability to absorb salt water was comparable to that of commercial gels.

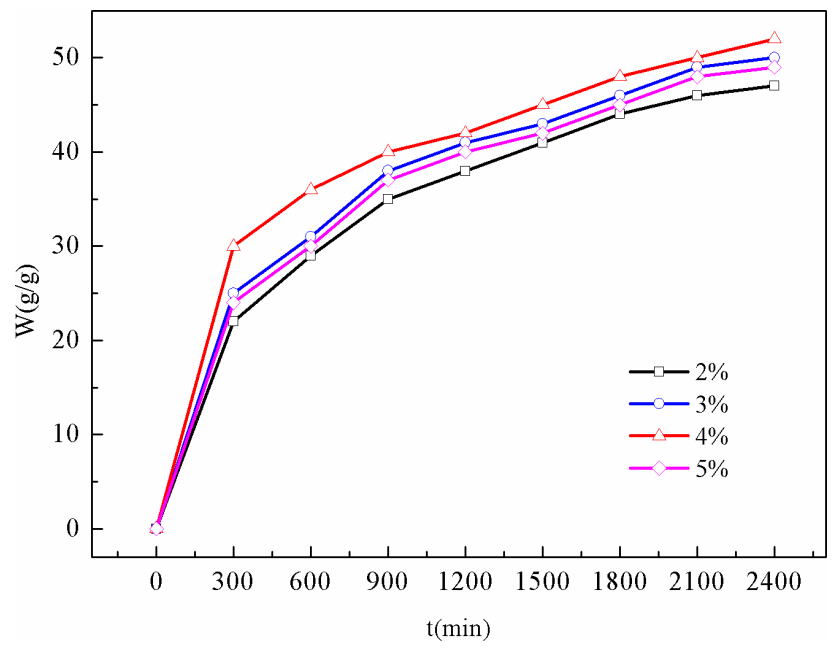

Figure 3: The brine absorption of foam gel at different SDS concentrations 
Table 1: Factors and Levels for Orthogonal Experimental Design

\begin{tabular}{cccc}
\hline Number & DAMCC (wt\%) A & $\begin{array}{c}\text { CMCS (wt\%) } \\
\text { B }\end{array}$ & $\begin{array}{c}\text { SDS (wt\%) } \\
\text { C }\end{array}$ \\
\hline 1 & $1 \%$ & $2 \%$ & $3 \%$ \\
2 & $2 \%$ & $3 \%$ & $4 \%$ \\
3 & $3 \%$ & $4 \%$ & $5 \%$ \\
\hline
\end{tabular}

Table 2: Orthogonal experimental results and range analysis

\begin{tabular}{ccccc}
\hline Number & A & $B$ & $C$ & W $(\mathrm{g} / \mathrm{g})$ \\
\hline $\mathrm{G}-1$ & 1 & 1 & 1 & 74 \\
$\mathrm{G}-2$ & 1 & 2 & 2 & 53.7 \\
$\mathrm{G}-3$ & 1 & 3 & 3 & 68.5 \\
$\mathrm{G}-4$ & 2 & 1 & 2 & 95 \\
$\mathrm{G}-5$ & 2 & 2 & 3 & 75 \\
$\mathrm{G}-6$ & 2 & 3 & 1 & 38.3 \\
$\mathrm{G}-7$ & 3 & 1 & 3 & 50.3 \\
$\mathrm{G}-8$ & 3 & 2 & 1 & 83.8 \\
$\mathrm{G}-9$ & 3 & 3 & 2 & 100 \\
\hline $\mathrm{K}_{1}$ & 65.4 & 73.1 & 65.3 & \\
$\mathrm{~K}_{2}$ & 69.4 & 70.8 & 82.9 & \\
$\mathrm{~K}_{3}$ & 78 & 68.9 & 64.6 & \\
\hline $\mathrm{R}_{j}$ & 12.6 & 4.2 & 18.3 & \\
\hline
\end{tabular}

\subsubsection{Water retention analysis}

Figure 4 presents the effect of different SDS concentrations on the water retention properties of foam gel. As indicated by the figure, the water retention turned optimal at $4 \%$ SDS. The reduction of water retention over time was decel-

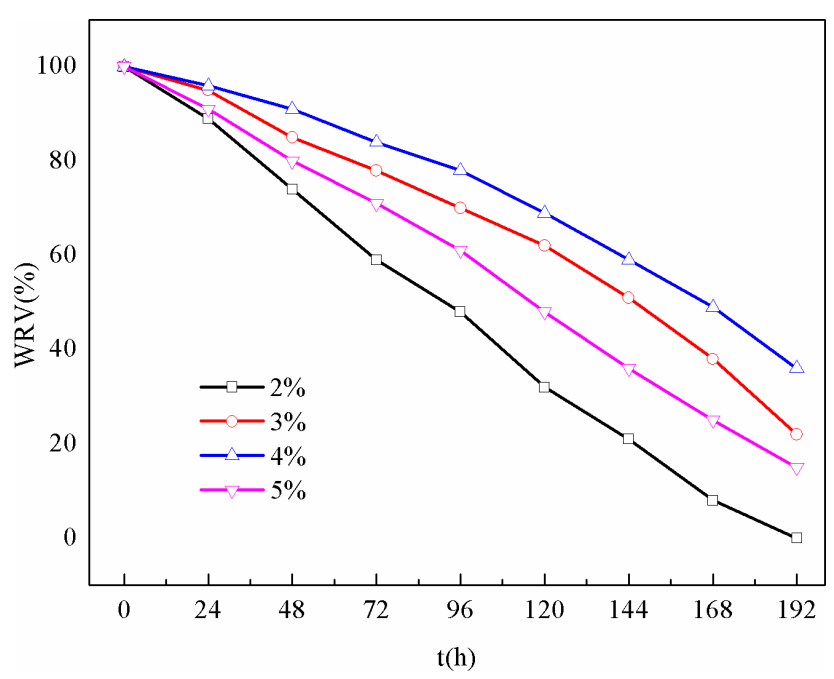

Figure 4: Water retention properties of foam gel at different SDS concentrations erated. To be specific, the time required for its water retention to drop to $50 \%$ was $168 \mathrm{~h}$. The foam gel could relatively long remain the integrity in water. In agriculture, excessive of watering will significantly impact soil erosion and compaction, thereby destroying the soil aggregate structure, which hinders the improvement of soil water and fertilizer retention. The good water retention properties of foam gel are capable of significantly reducing the frequency of watering and improve soil water and fertilizer conservation. In addition, foam gel is expected to become a novel type of soil amendment.

\subsection{Foam stability of foam gel}

The foam stability was characterized by height difference before and after the physical foaming. Figure 5 shows that under the foaming agent (SDS) concentration of $4 \%$, after static $190 \mathrm{~h}$, the height of the foam gel varied slightly, and no bleeding phenomenon was identified. This resulted from the cross-linking effect between the gels, which could significantly lock the vesicles, leading to high stability and difficulty in collapse. Moreover, the high compatibility between cellulose and chitosan can also account for the significant foam stability of foam gel.

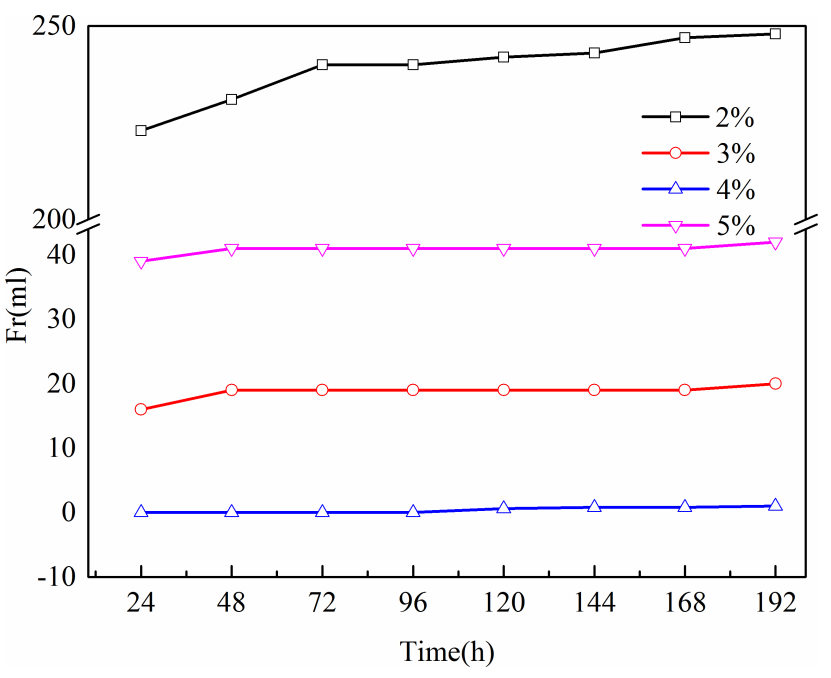

Figure 5: Foam stability analysis

\subsection{Re-expansion performance of foam gel}

Figure 6 presents the rewetting behavior of the foam gel after 4 swelling cycles in deionized water. It is suggested that the water absorption of foam gel maintained a high 
level after several sorption-desorption cycles. To be specific, the water absorption capacity of foam gel decreased by $5 \%$ in the first three cycles. This result was attributed to the hydrogen bonding between carboxyl group and hydroxyl group, thereby reducing its ability to adsorb water. After the 4th cycle, the re-expansion behavior of foam gel turned out to be comparable to that of the $3^{\text {rd }}$ cycle, and the water adsorption rate stopped decreasing. Afterwards, the results indicated that the foam gel exhibited effective water absorption and re-expansion properties. Such high reusability of foam gel makes it a good candidate for its application as water absorbing agent.

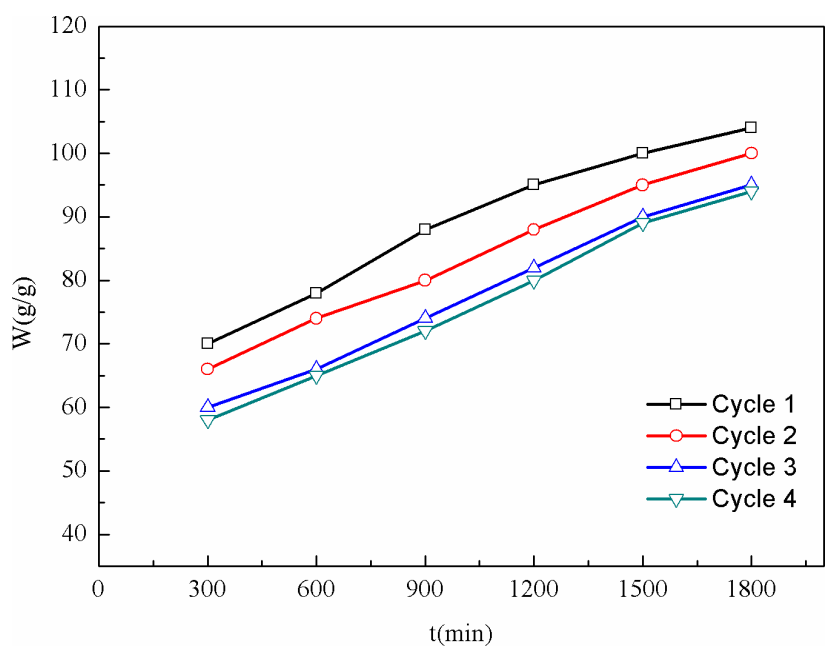

Figure 6: Reflux properties of foam gel

\subsection{Morphology of foam gel}

Figure 7 illustrates the SEM images of DAMCC/CMCS foam gel material synthesized at $4 \%$ SDS. The sample showed a large network structure, the pores were interconnected, and the pore diameter reached 350-450 $\mu \mathrm{m}$. In addition, flaky walls and ultrathin structure were observed in the foam gel. The size and shape of pores could be maintained during the crosslinking, and SDS molecules could be dispersed uniformly throughout the hydrogel polymer.

As mentioned above, foam gel exhibits effective water absorption performance. This is explained below. First, foam is evenly distributed inside the gel, allowing it to absorb water sufficiently. Second, the aldehyde group on the DAMCC surface reacts with the free amino group on the CMCS macromolecular chain to generate a Schiff base reaction, which can immobilize the foam. Thus, the water absorption of foam gel is significantly improved by its polymer network structure.

\subsection{FTIR analysis}

FTIR spectra of DAMCC/CMCS ranging from 4000 to $400 \mathrm{~cm}^{-1}$ are presented in Figure 8. Compared with MCC, DAMCC displays characteristic absorption peaks of aldehyde group (-CHO) at $1718 \mathrm{~cm}^{-1}$ and $885 \mathrm{~cm}^{-1}$. The former pertains to the stretching vibration of free aldehyde group, and the latter belongs to the vibration of semiacetal. The aldehyde group synthesized in the reaction rarely existed as free aldehyde. As indicated from the results, $\mathrm{NaIO}_{4}$ destroyed the carbon-carbon bond on the glucose ring of MCC, and the hydroxyl group was partially oxidized to aldehyde group, thereby leading to the production of DAMCC [29]. The absorption peaks of CS at $1653 \mathrm{~cm}^{-1}$ and $1420 \mathrm{~cm}^{-1}$ respectively belong to the asymmetrical tensile vibration and symmetric tensile vibration of COO-. The absorption peaks of modified (CMCS) shift. Compared with DAMC, the corresponding aldehyde group absorption peak at $1718 \mathrm{~cm}^{-1}$ is no longer identified in the foam gel spectrum. As revealed from the results, Schiff base reaction between the amino group on surface and the aldehyde group was generated, which complies with the study by Wei et al. [30].

\subsection{XRD analysis}

XRD spectra of DAMCC/CMCS are presented in Figure 9. There was an obvious crystal zone in MCC at $2 \theta=15.5^{\circ}$ and $22.7^{\circ}$. When MCC was oxidized, the intensity of its diffraction peak decreased, and the crystallinity was reduced, demonstrating that the oxidation of sodium periodate resulted in more amorphous MCC and destroyed the crystallized area of MCC [31]. CS refers to a type of semicrystalline polysaccharide displaying two major diffraction peaks at $2 \theta=10^{\circ}$ and $20^{\circ}$ [32]. Notably, CMCS completely altered the typical structure of $\mathrm{CS}$, and a new diffraction peak was identified at $2 \theta=32.4^{\circ}$ and $45.4^{\circ}$, leading to the formation of an amorphous material. In the foam gel, as impacted by the addition of SDS and the Schiff base reaction, the crystal structure of MCC was fully destroyed, and the crystallinity of CMCS was reduced. 

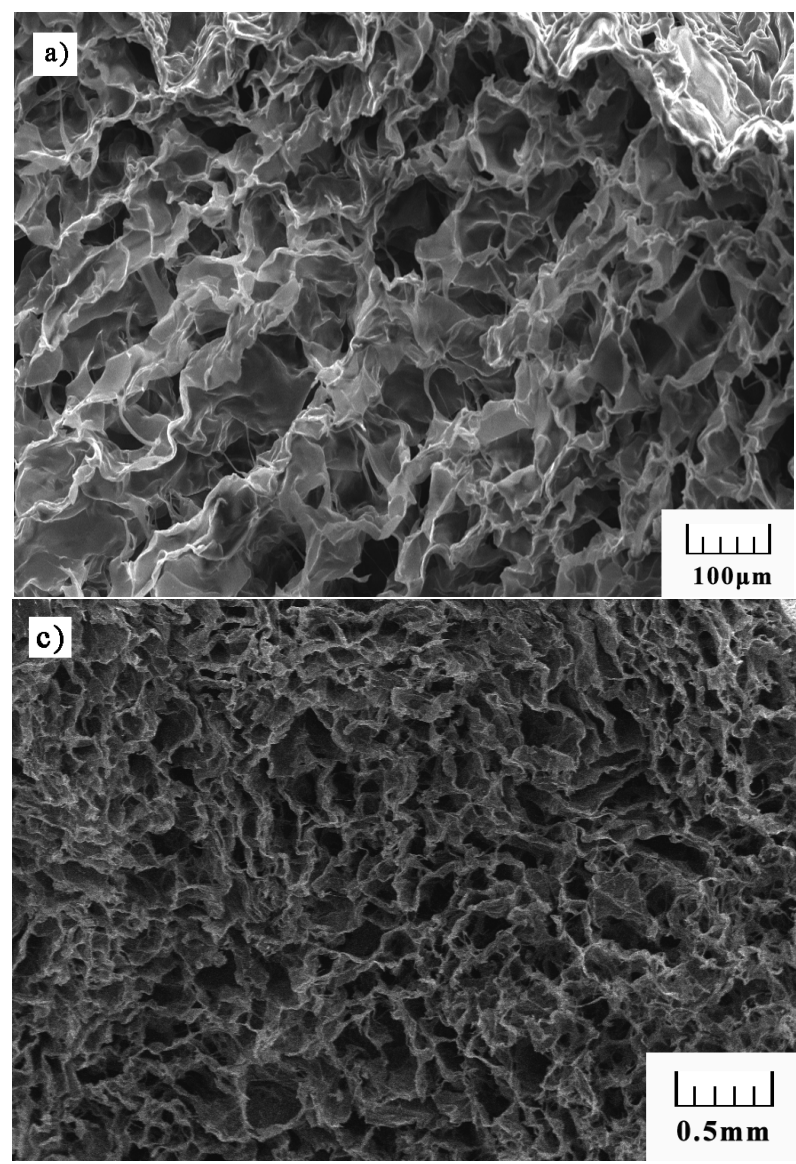

$0.5 \mathrm{~mm}$
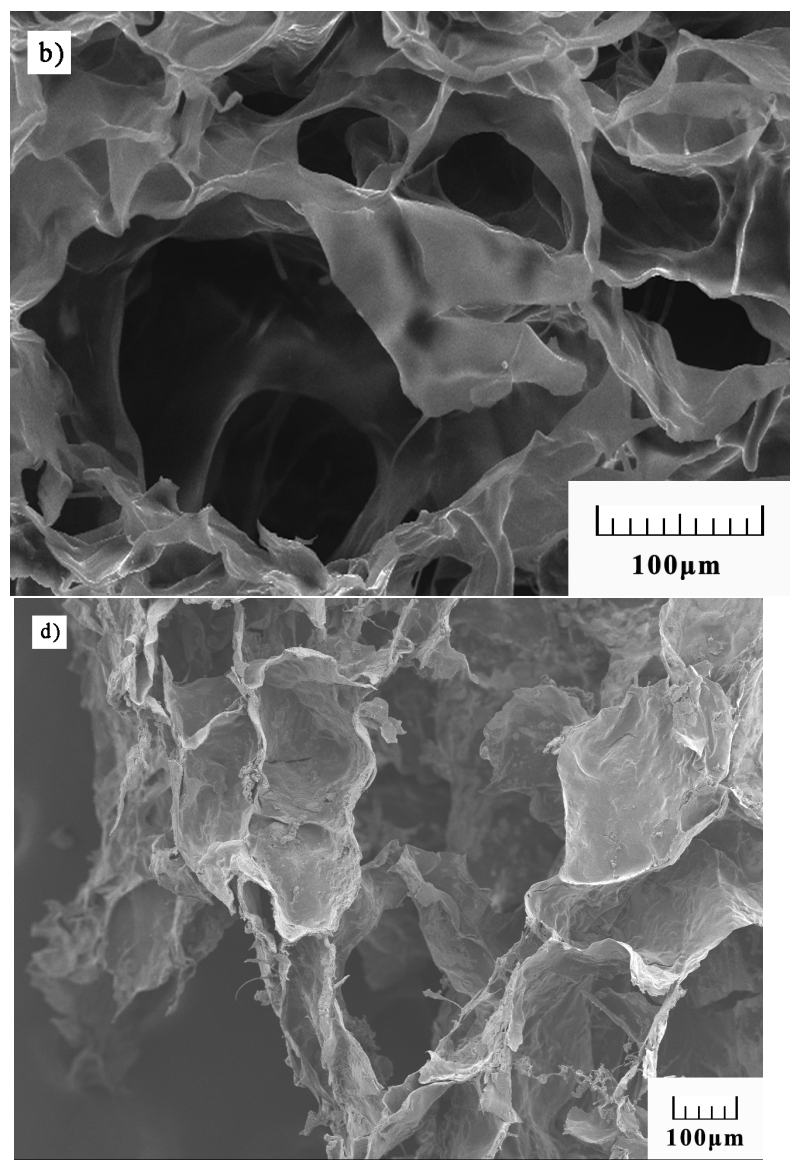

Figure 7: SEM images of foam gel synthesized at $4 \%$ SDS

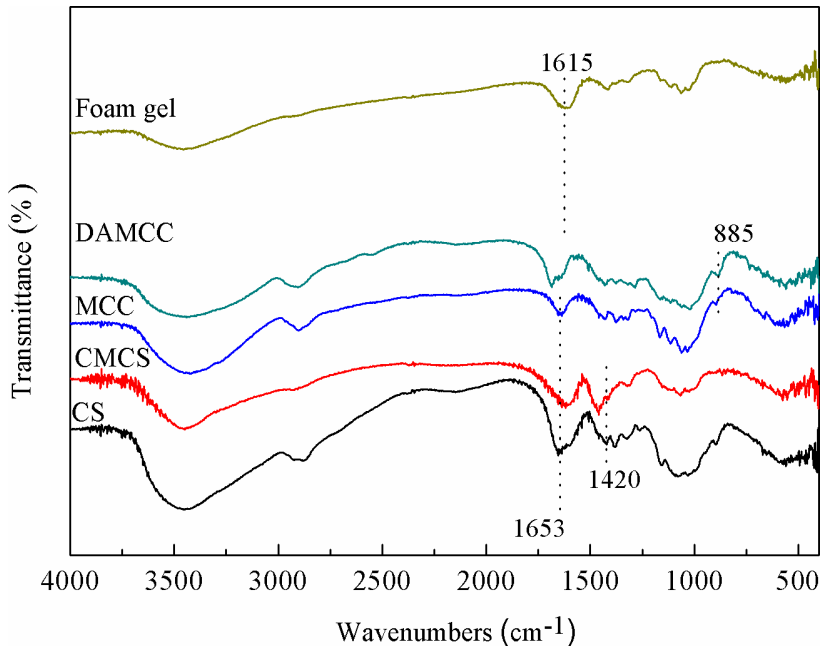

Figure 8: FTIR spectra of CS, CMCS, MCC, DAMCC and DAMCC/CMCS foam gel

\subsection{BET analysis}

The adsorption desorption isotherm of the absorbent material is illustrated in Figure 10. Given the type of the ad-

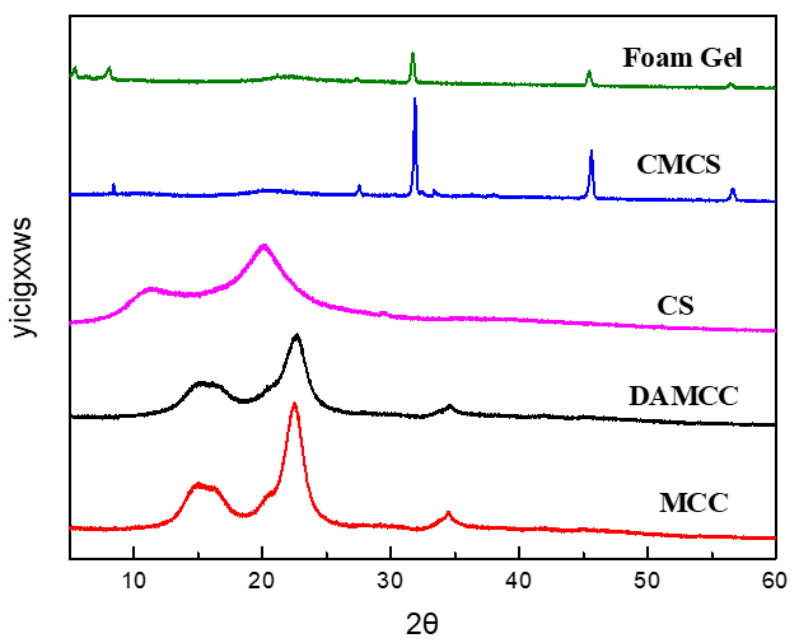

Figure 9: XRD images of CS, CMCS, MCC, DAMCC and DAMCC/CMCS foam gel

sorption desorption curve, the curve belongs to the V-type isotherm, and the adsorption capacity increased gradually with the increase in pressure. The adsorption was similar to that of the macroporous material, demonstrating 
that the material exhibited a macroporous structure. However, due to capillary, the adsorption capacity of foam gel showed a steep increase at high pressure, attributed to capillary condensation. At the highest point, the pores were filled, and the adsorption isotherm reached equilibrium. In the desorption, capillary condensation and capillary evaporation did not occur under the identical pressure, causing the generation of hysteresis ring. It can be concluded that the hysteresis loop did not reach equilibrium under the relative pressure comparable to the saturated vapor pressure, demonstrating that the pores between the materials were formed by the slit between the mixtures. Moreover, the above figure shows that the starting point of the hysteresis loop occurred between 0.8 and 1 , leading to the existence of many and dense pores in the material. On the one hand, the existence of large pores in the material could result from the pore structure formed by water sublimation in the vacuum freeze-drying. On the other hand, it can be attributed to the pressure difference between the fibers in the vacuum environment; thus, the material tends to form larger pores.

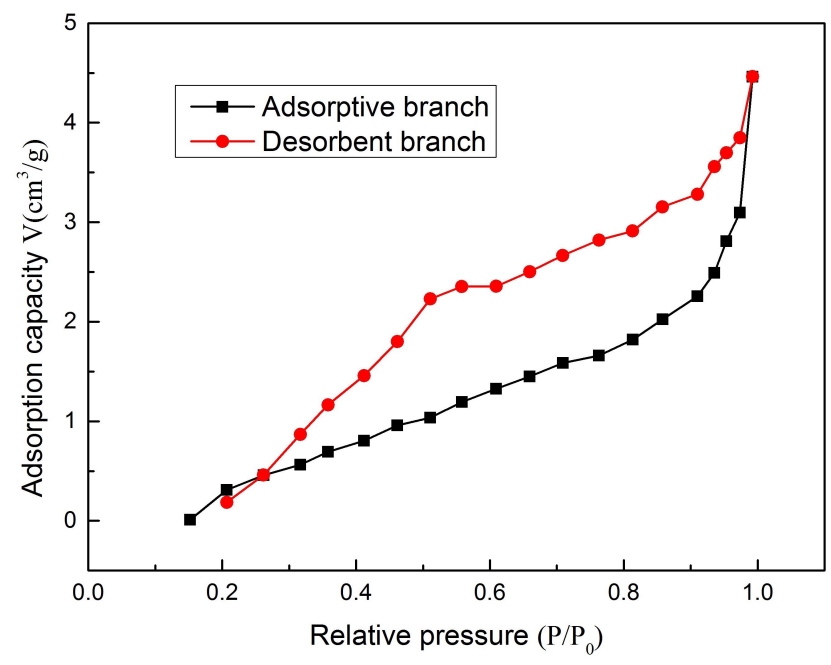

Figure 10: BET images of DAMCC/CMCS foam gel

\section{Conclusions}

In the present study, a new type of DAMCC/CMCS foam gel water absorbent was synthesized without any catalyst, exhibiting both economic and environmental advantages. By performing a series of experiments, the following conclusions were drawn. Under the DAMCC content of 3\%, the CMCS content of $2 \%$ and the SDS concentration of $4 \%$, the optimal performances of foam gel (e.g., water absorption, water retention, foam stability and re-expansion behavior) were achieved. The morphology study revealed a large network structure of foam gel, long maintaining its integrity in water. The foam gel synthesized in this study exhibited high adsorption capacity as well as prominent stability and reusability. It is expected to replace pure cellulose absorbent materials and partial commercial gels, and it is reported with suitable applications in health, pharmaceutical, food and agricultural manufacturing industries.

Author Contributions: Conceptualization, Hongkai Zhao and Kehan Zhang; Investigation, Hongkai Zhao and Kehan Zhang; Methodology, Hongkai Zhao, Kehan Zhang,Shoupeng Rui and Peipei Zhao; Validation, Hongkai Zhao and Kehan Zhang; Writing-original draft, Hongkai Zhao and Kehan Zhang; Writing-review and editing, Hongkai Zhao and Kehan Zhang.

Acknowledgement: The authors would like to thank the financial support provided by the National key point research and invention program of the thirteenth (Grant No. 2018YFD1101001), and supported by the 13nd 5-year Science and Technology Research Program of the Department of Education of Jilin Province (No. JJKH20170239KJ).

Conflict of Interests: The authors declare no conflicts of interest.

\section{References}

[1] J. Tang, X.-X. Tang, Y.-M. Qin, Q.-S. He, Y. Y, Z.-L. Ji, Karst rocky desertification progress: Soil calcium as a possible driving force, Sci Total Environ. 2019,649,1250-1259.

[2] F. Wahid, X.-H. Hu, L.-Q. Chu, S.-R. Jia, Y.-Y. Xie, C. Zhong, Development of bacterial cellulose/chitosan based semiinterpenetrating hydrogels with improved mechanical and antibacterial properties, J. Int Biol Macromol, 2018,18,0140-8130.

[3] U.-G. Sampath, Y.-C. Ching, C.-H. Chuah, R. Singh, P.-C. Lin, Preparation and characterization of nanocellulose reinforced semiinterpenetrating polymer network of chitosan hydrogel, J. Cellulose, 2017,5,2215-2228.

[4] Y.-M. Zhou, S.-Y. Fu, L.-L. Zhang, H.-Y. Zhan, M.-V. Levit, Use of carboxylated cellulose nanofibrils-filled magnetic chitosan hydrogel beads as adsorbents for Pb (II), J. Carbohyd polym, 2014,101,7582.

[5] L.-H. Fan, J. Yang, H. Wu, Z.-H. Hu, J.-Y. Yi, J. Tong, X.-M. Zhu, Preparation and characterization of quaternary ammonium chitosan hydrogel with significant antibacterial activity, J. Int biol Sci, 2015,79,830-836.

[6] M. Alam, M. Islam, L. Christopher, Sustainable Production of Cellulose-Based Hydrogels with Superb Absorbing Potential in 
Physiological Saline, ACS Omega. 2019,4,9419-9426.

[7] D. Zhang, L. Wang, H.-H. Zeng, P. Yan, J. Nie, V. Sharma, C.-Y. Wang, A three-dimensional macroporous network structured chitosan/cellulose biocomposite sponge for rapid and selective removal of mercury(II) ions from aqueous solution, Chem. Eng. J. 2019,19,796-807.

[8] M. He, H. Chen, X.-J. Zhang, C.-S. Wang, C. Xu, Y.-T. Xue, J.-S. Wang, P.-H. Zhou, Q.-X. Zhao, Construction of novel cellulose/chitosan composite hydrogels and films and their applications, Cellulose. 2018,42,1130-1141.

[9] J. Stratz, A. Liedmann, M. Trutschel, K. Mader, T. Groth, S. Fischer, Development of hydrogels based on oxidized cellulose sulfates and carboxymethyl chitosan, Cellulose. 2019,20,1251-1260.

[10] S. Ramachandran, S. Nandhakumar, M. Dhanaraju, Formulation and Characterization of Glutaraldehyde Cross-Linked Chitosan Biodegradable Microspheres Loaded with Famotidine, Trop. J. Pharm. Res. 2011,10,309-316.

[11] K. Abdul, C. Saurabh, A. Adnan,M. Fazita, M. Syakir, Y. Davoudpour, M. Rafatullah, C. Abdullah, M. Haafiz, R. Dungani, A review on chitosan-cellulose blends and nanocellulose reinforced chitosan biocomposites: Properties and their applications, Carbohyd. Polym. 2016,150,216-226.

[12] X.-J. Zhou, H. Essawy, M. Mohamed, H. Ibrahim, N. Ammar, Grafting polymerization of acrylic acid onto chitosan-cellulose hybrid and application of the graft as highly efficient ligand for elimination of water hardness:Adsorption isotherms, kinetic modeling and regeneration, Chem. Eng.J. 2018,6,2137-2147.

[13] F. Cheng, J. He, T.-S. Yan, C.-Y. Liu, X.-J. Wei, J.-W. Li, Y.-D. Huang, Antibacterial and hemostatic composite gauze of N,Ocarboxymethyl chitosan/oxidized regenerated cellulose, RSC Adv. 2016,56,1039-1048.

[14] A. Teotia, S. Ikram, B. Gupta, Structural characterization of chitosan and oxidized carboxymethyl cellulose based freeze-dried films, Polym. Bull. 2012,69,175-188.

[15] Q. Zhao, J.-W. Qian, Q.-F. An, C.-J. Gao, Z.-L. Gui, H.-T. Jin, Synthesis and characterization of soluble chitosan/sodium carboxymethyl cellulose polyelectrolyte complexes and the pervaporation dehydration of their homogeneous membranes, J. Membrane. Sci. 2009,333,68-78.

[16] F. Hafida, N. Aouaz, N. Dairi, Environmental-sensitive chitosan-gpolyacrylamide/carboxymethylcellulose superabsorbent composites for wastewater purification I: synthesis and properties, Polym. Bull. 2016,73,815-840.

[17] B.-B. Wang, X.-D. Yang, C.-D. Qiao, Y. Li, T.-D. Li, C.-L. Xu, Effects of chitosan quaternary ammonium salt on the physicochemical properties of sodium carboxymethyl cellulose-based films, Carbohyd. Polym. 2017,184,37-48.

[18] G. Antova, P.Vasvasova, M. Zlatanov, Studies upon the synthesis of cellulose stearate under microwave heating, Carbohyd. Polym. 2004,57,131-134.

[19] S. Kamel, N. Ali, K. Jahangir, S.-M. Shah, A. Gendy, Pharmaceutical significance of cellulose:A review, Express Polym. Lett. 2008,2,758-778.
[20] S. Liu, G. Sun, Radical graft functional modification of cellulose with allyl monomers: Chemistry and structure characterization, Carbohyd. Polym. 2008,71,614-625.

[21] H. Essawy, M. Ghazy, F. El-hai,M. Mohamed, Superabsorbent hydrogels via graft polymerization of acrylic acid from chitosancellulose hybrid and their potential in controlled release of soil nutrients, Int. J. Biol. Macromol. 2016,89,144-151.

[22] S.-W. Zhang, W.-T. Liu, J. Liang, X.-Y. Li, W.-N. Liang, S.-Q. He, C.-S. Zhu, L.-Y. Mao. Buildup mechanism of carboxymethyl cellulose and chitosan self-assembled films, Cellulose. 2013,20,11351143.

[23] K. Kanimozhi, S. Basha, V. Kumari, K. Kaviyarasu, Development of Biomimetic Hybrid Porous Scaffold of Chitosan/Polyvinyl Alcohol/Carboxymethyl Cellulose by Freeze-Dried and Salt Leached Technique, J. Nanosci. Nanotechno. 2018,18,4916-4922.

[24] Z.-N. Cao, X.-G. Luo, H. Zhang, Z. Fu, Z. Shen, N. Cai, Y.-N. Xue, F.-Q. Yu, A facile and green strategy for the preparation of porous chitosan-coated cellulose composite membranes for potential applications as wound dressing, Cellulose. 2016,23,1349-1361.

[25] L.-H. Fan, C. Tan, L.-B. Wang, X.-R. Pan, M. Cao, F. Wen, W.G. Xie, M. Nie, Preparation, characterization and the effect of carboxymethylated chitosan-cellulose derivatives hydrogels on wound healin, J. Appl. Polym. Sci. 2013,128,492-507.

[26] S. Borysiak, A. Grząbka-Zasadzinska, Influence of the polymorphism of cellulose on the formation of nanocrystals and their application in chitosan/nanocellulose composites, J. Appl. Polym. Sci. 2015,133,1760-1765.

[27] S.-F. Yan, J.-B. Yin, L. Tang, X.-S. Chen, Novel physically crosslinked hydrogels of carboxymethyl chitosan and cellulose ethers:Structure and controlled drug release behavior, J. Appl. Polym. Sci. 2011,119,2350-2358.

[28] A. Errokh, A. Magnin, J.-L. Putaux, S. Boufi, Morphology of the nanocellulose produced by periodate oxidation and reductive treatment of cellulose fibers, J. Cellulose, 2018,7,3899-3911.

[29] M.- N. Alam, and L. Christopher, Natural Cellulose-Chitosan Crosslinked Superabsorbent Hydrogels with Superior Swelling Properties, J. ACS Sustain Chem Eng, 2018.

[30] D.-D. Wei, Q.-X. Liu, Z.-D. Liu, J. Liu, X.-J. Zheng, Y. Pei, K.-Y. Tang, Modified nano microfibrillated cellulose/carboxymethyl chitosan composite hydrogel with giant network structure and quick gelation formability, J. Int. J. Biol. Macromol. 2019,135,561568.

[31] E.-S. Madivoli, P.-G. Kareru, A.-N. Gachanja, S.-M. Mugo, D.-S. Makhanu, Synthesis and characterization of dialdehyde cellulose nanofibers from 0. sativa husks, J. SN Appl Sci, 2019,7,723.

[32] P. Guerrero, A. Muxika, I. Zarandona, K. Caba, Crosslinking of chitosan films processed by compression molding, Carbohyd Polym, 2018. 